\title{
Increased sensitivity and field of view for a rotating positron camera
}

\author{
R Clack $\dagger, \mathrm{D}$ Townsend $\ddagger$ and A Jeavons $\dagger$ \\ † EP Division, CERN, Geneva, Switzerland \\ $\ddagger$ Division of Nuclear Medicine, Cantonal Hospital, Geneva, Switzerland
}

Received 16 March 1984, in final form 4 July 1984

\section{Introduction}

Large area positron cameras for three-dimensional imaging in medicine have been under development for several years at a number of centres (McKee et al 1979, Bateman et al 1980, Jeavons et al 1981, Del Guerra et al 1983), and some groups have reported clinical applications (Ott et al 1983, Townsend et al 1984). To reconstruct the data, an algorithm based on a backprojection and frequency space deconvolution approach (Chu and Tam 1977, Schorr and Townsend 1981, Schorr et al 1983) has been adopted by each group. Such deconvolution methods have the advantage of being computationally efficient but require that the point response function be shift invariant (Chu and Tam 1977). In this context, the point response function is the backprojected image of a unit point source of activity and 'shift invariance' means that displacing the source causes no change in the point response function other than an identical displacement. For a stationary, dual detector camera, shift invariance is ensured by rejecting positron annihilation event-lines (figure 1) with directions that fall outside specific angular

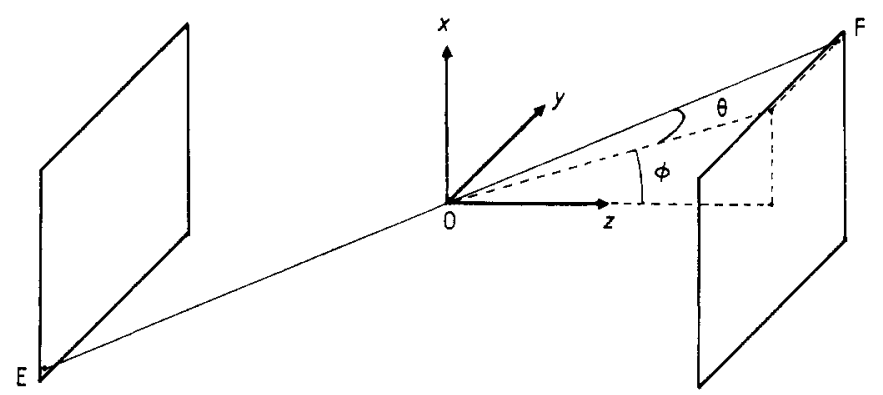

Figure 1. Angles $\theta$ and $\phi$ defined for a specific positron annihilation event line (EF).

limits (Schorr and Townsend 1981) (figure 2a). Traditionally, the acceptance region for a point source is referred to as a cone, although for square or rectangular detectors, the shape of the acceptance region is actually that of a pyramid. However, even with angular restrictions, the response function is shift invariant only for points that lie within a limited region or field of view (figure $2 b$ ). The angles of the acceptance cone may be calculated from the size and separation of the detectors and the size of the 


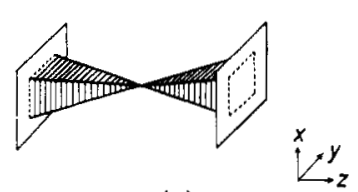

(a)

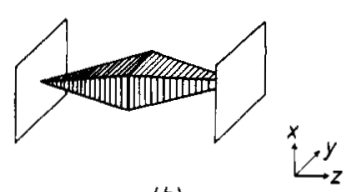

(b)

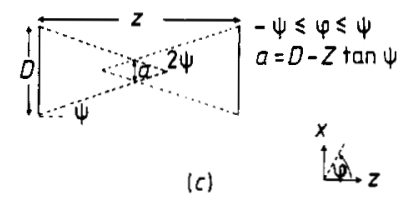

(c)

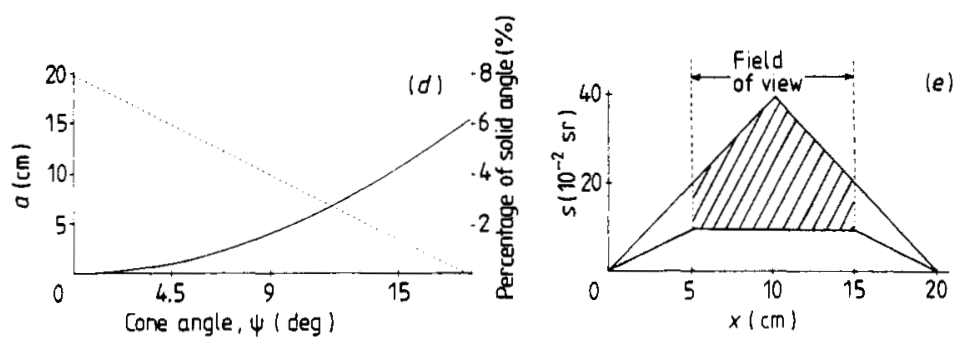

Figure 2. (a) Acceptance cone for a point source of activity placed midway between the detectors. (b) Allowable field of view of the system with a cone of constraint corresponding to the cone in $(a)$. (c) Relationship between the size of the field of view in the midplane $a$ and the angle of the cone $\psi$. Square detectors of side $D$ are separated by a distance $Z$. (d) Maximum size of the field of view (---) and the percentage of the total solid angle covered (_- ) as a function of the angle of the cone (for a pyramid of square cross-section); $D=20 \mathrm{~cm}, Z=61 \mathrm{~cm}$. (e) Profile through the centre of the plane midway between a pair of stationary detectors. The curves show the acceptance as a function of distance across the plane. Upper curve is without a cone of constraint, lower curve is for a cone which gives a uniform field of view of $10 \mathrm{~cm} \times 10 \mathrm{~cm}$. The shaded region shows the events rejected by the cone. The vertical scale $s$ is in one-hundredths of steradians; $D=20 \mathrm{~cm}, Z=61 \mathrm{~cm}$.

required field of view-the larger the field of view, the smaller the constraint angles and hence the greater the fraction of rejected events (figure $2 c$ ). A compromise is necessary between the field of view and the event rejection fraction to maintain adequate system sensitivity (figure $2 d$ ). For a stationary camera, a typical field of view of one half the detector size results in rejection of $50 \%$ to $75 \%$ of the data (figure $2 e$ ).

To overcome the data reconstruction problems associated with a limited-angle stationary camera, rotation of either the detectors (Townsend et al 1983) or the patient is required to obtain the necessary additional projections. Rotation may be performed in discrete angular steps (e.g. six steps of $30^{\circ}$ ) and the data reconstructed by treating each angular position as an independent, limited-angle system (figure $3 a$ ) (Townsend et al 1983). The same cone of constraint with respect to a coordinate system that rotates with the detectors is applied at each angular position, and the actual angle of the camera in a fixed coordinate system is taken into account when summing the individual backprojections. Shift invariance of the overall system is then a consequence of the shift invariance of each individual angular position. The angular limits of the cone determine the dimensions of the field of view (figure $3 b$ ). With this approach, enlarging the field of view decreases the system sensitivity in the same way as it does for a stationary camera.

The decrease in sensitivity may be avoided by relaxing the requirement of shift invariance for each angular position of the detectors. In practice, the cone constraint is applied as independent angular limits on $\phi$ and $\theta$. Dispensing entirely with the constraint on the angle $\phi$, shift invariance of the overall response function can be re-established using the techniques detailed in $\$ 2$, even though shift invariance is lost in the individual angular positions. Of perhaps greater importance than the 

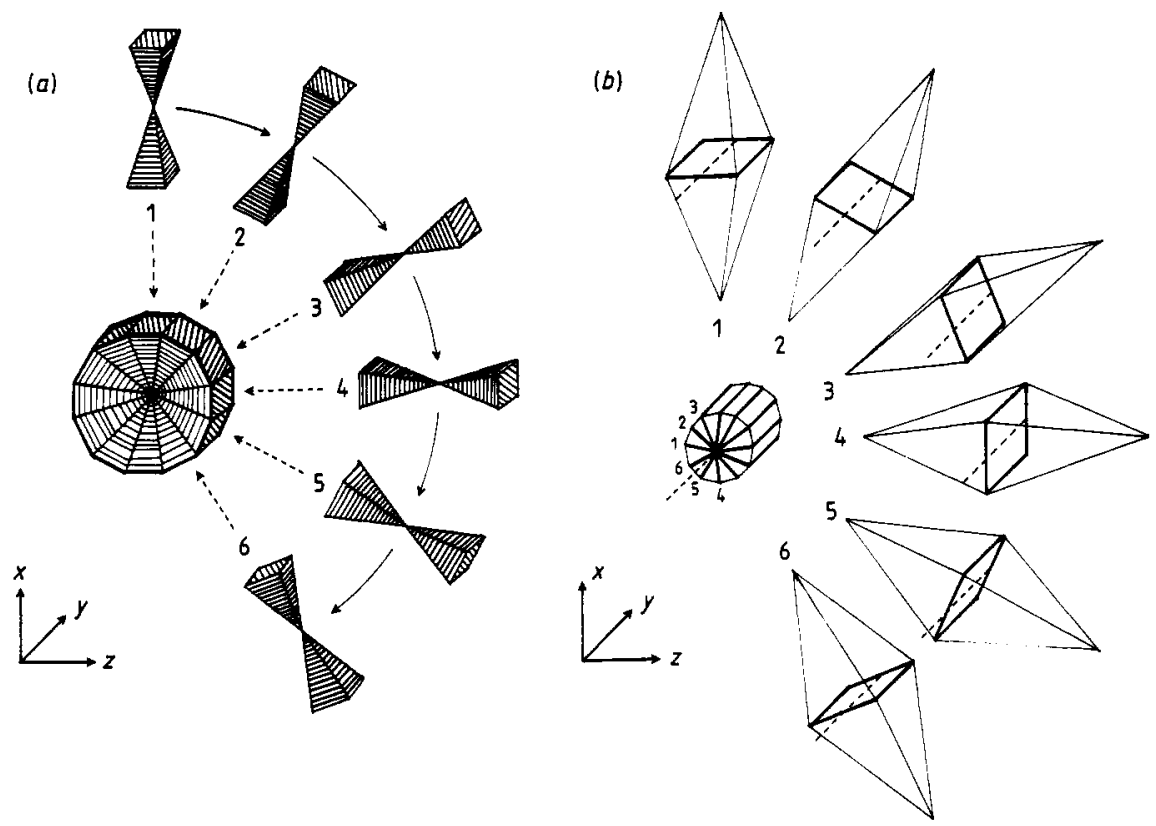

Figure 3. (a) Same acceptance cone as in figure 2(a) applied to each of six angular positions of the detectors which are summed to give the support of the overall point response function. (b) Six fields of view corresponding to the cones in $(a)$; the overall field of view is the intersection of the six individual fields. The rotation axis is shown as the dotted line.

increase in sensitivity is the fact that, with the angle $\phi$ limited only by the geometrical acceptance of the detectors, the sensitivity is no longer coupled to the diameter of the field of view.

This paper will describe two different approaches for the reconstruction of data from a rotating, area detector, positron camera, without the need to constrain $\phi$. The constraint on the angle $\theta$ remains. As will be seen, there is a considerable increase in the overall sensitivity and usable field of view of the camera.

\section{Elimination of the constraint on $\phi$}

The point response function for each angular position of the detectors has a pyramid shaped support, even in the absence of a $\phi$ constraint when the rectangular base is defined in the $\phi$ direction by the geometrical acceptance of the detectors, and in the $\theta$ direction by the usual angular constraint. Although different points within the field of view will have different geometrical acceptances in $\phi$, the overall response function will have the same support as in figure $3(a)$ if, for every point, the geometrical acceptances in each angular position overlap, or at least touch, those in the adjacent angular position. The functional behaviour inside the support will, however, differ for different points, but by compensating for these variations shift invariance can be restored.

The size of the field of view is determined by the distance between the centre of rotation and the point where adjacent geometrical acceptances just touch. Reducing the rotational step size increases the field of view, without affecting sensitivity. The height of the field of view is limited by the $\theta$ constraint, as usual. 
Two different methods of dealing with the functional variations in the overall point response function are presented here. The first involves a modification to the backprojection procedure, and the second scales the image after reconstruction; neither method significantly increases the computational load.

\subsection{Weighted backprojection}

The first scheme assigns to each coincidence channel a specific weight by which to multiply the value normally backprojected. A coincidence channel is an event line within the acceptance of the detectors, and with weighted backprojection, weights are calculated according to the time the channel spends within the detector acceptance during rotation. For continuous rotation the weights are a function only of the distance of the channel from the axis of rotation (figures $4 a, b$ ). The value of the weight for a particular channel is given by the reciprocal of the total time the channel spends inside the geometric acceptance, normalised such that unit weight is assigned to channels outside and tangential to the edge of the field of view (figure $4 c$ ). For channels intersecting the field of view, all weights are less than unity, with the minimum weight decreasing as the field of view is enlarged. As an example, for a field of view of diameter $90 \%$ of the length of the detector face, the minimum weight is approximately 0.1 .
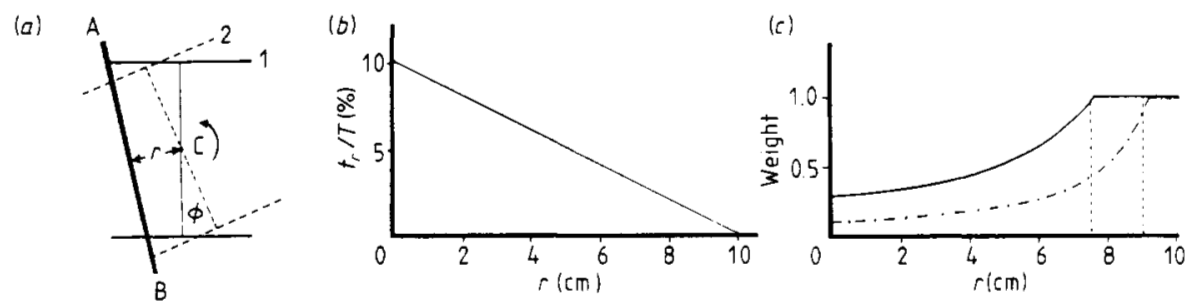

Figure 4. (a) A coincidence channel AB shown for two positions $(1,2)$ of the detectors; $r$ is the perpendicular distance of the channel from the centre of rotation. $T$ is the total time for a complete rotation. $t_{\mathrm{r}}=(\phi / \pi) T$. (b) The time $t_{\mathrm{r}}$ (as a percentage of the total time $T$ ) that each coincidence channel is seen by the detectors as a function of the distance $r$ from the centre of rotation. A continuous rotation of the detectors is assumed. $D=20 \mathrm{~cm} ; Z=61 \mathrm{~cm}$. (c) The backprojection weights (normalised) plotted as a function of $r$ for a continuously rotating camera. The curves are for fields of view of diameters $\longrightarrow, 15 \mathrm{~cm}$; and $-\cdot-, 18 \mathrm{~cm}$.

The weighting scheme ensures that, for a complete rotation, all channels intersecting a point within the field of view respond equally to activity at that point. Furthermore, the response to a point source is the same at all positions within the field of view, thereby ensuring shift invariance. Fourier deconvolution may then be performed in the usual way, with the filter given in Schorr et al (1983).

\subsection{Post-reconstruction scaling}

An alternative approach to the weighted backprojection procedure described above is to ignore the loss of shift invariance of the response function until after the backprojection and Fourier deconvolution have been completed. Backprojection is performed as usual, but with no constraint placed on the angle $\phi$. The resultant backprojection image $g_{1}(x, y, z)$ is therefore related to the unknown distribution of positron activity 
$f(x, y ; z)$ by the integral equation

$$
g_{1}(x, y, z)=\iiint f\left(x^{\prime}, y^{\prime}, z^{\prime}\right) h_{1}\left(x^{\prime}, y^{\prime}, z^{\prime}, x, y, z\right) \mathrm{d} x^{\prime} \mathrm{d} y^{\prime} \mathrm{d} z^{\prime}
$$

where $h_{1}\left(x^{\prime}, y^{\prime}, z^{\prime}, x, y, z\right)$ is the shift variant point response function (Jeavons et al 1981). An analysis of the function $h_{1}$ shows that it may be approximated by

$$
h_{1}\left(x^{\prime}, y^{\prime}, z^{\prime}, x, y, z\right) \cong s\left(x^{\prime}, y^{\prime}, z^{\prime}\right) h\left(x-x^{\prime}, y-y^{\prime}, z-z^{\prime}\right)
$$

The function $h\left(x-x^{\prime}, y-y^{\prime}, z-z^{\prime}\right)$ is the shift invariant point response function that has been discussed in detail elsewhere (Schorr and Townsend 1981, Schorr et al 1983) and $s\left(x^{\prime}, y^{\prime}, z^{\prime}\right)$ is a scale factor defined in equation (5) below. Substituting equation (2) into equation (1) and setting $f_{1}\left(x^{\prime}, y^{\prime}, z^{\prime}\right)=f\left(x^{\prime}, y^{\prime}, z^{\prime}\right) s\left(x^{\prime}, y^{\prime}, z^{\prime}\right)$ gives

$$
g_{1}(x, y, z)=\iiint f_{1}\left(x^{\prime}, y^{\prime}, z^{\prime}\right) h\left(x-x^{\prime}, y-y^{\prime}, z-z^{\prime}\right) \mathrm{d} x^{\prime} \mathrm{d} y^{\prime} \mathrm{d} z^{\prime}
$$

The backprojection $g_{1}(x, y, z)$ differs from the backprojection distributions discussed in Schorr et al (1983) because it is related to $f(x, y, z)$ by a shift variant response function. Equation ( 3 ), however, is a convolution equation and may be solved by the Fourier convolution theorem as described in Schorr et al (1983) to give the function $f_{1}(x, y, z)$. The positron activity distribution $f(x, y, z)$ can then be obtained directly from

$$
f(x, y, z)=f_{1}(x, y, z) / s(x, y, z)
$$

For continuous rotation, the scale factors $s(x, y, z)$ are always positive and depend only upon the perpendicular distance of the point $(x, y, z)$ from the axis of rotation. Thus, if the centre of rotation in a given transverse plane is at $(0, y, 0)$, then the appropriate scale factor at the point $(x, y, z)$ is given by

$$
s(x, y, z)=c\left[\sqrt{ }\left(x^{2}+z^{2}\right)\right]=c(r)
$$

The function $c(r)$ is the same for all transverse planes and reflects the local increase in sensitivity that results from removing the constraint on the angle $\phi$. It may be calculated at each point in the plane by taking the ratio of the sensitivities with and without the constraint on $\phi$. Figure $5(a)$ is a hidden line display of $c(r)$ for a fixed

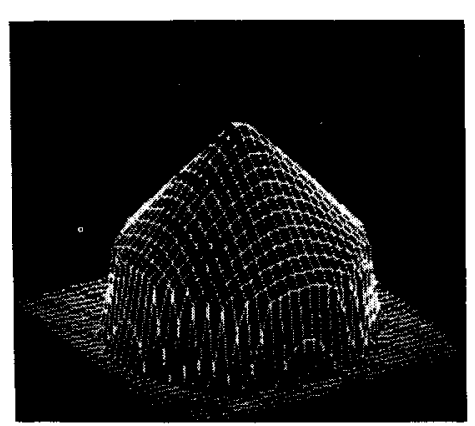

(a)

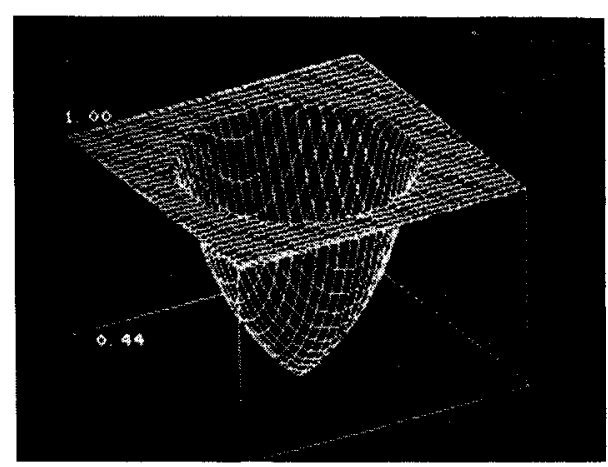

(b)

Figure 5. (a) Variation of sensitivity for a plane transverse to the axis of rotation. The sensitivity is set to zero for $r$ greater than $20 \mathrm{~cm}$ and the grid line spacing is $2 \mathrm{~mm}$. The peak value is inversely proportional to the difference between the diameter of the field of view and the detector size. (b) Factors used to scale each transverse plane after reconstruction for a field of view of diameter $18 \mathrm{~cm}$. 
transverse plane, where $c(r)=0$ for $r$ greater than $20 \mathrm{~cm}$. The vertical scale depends on the diameter of the field of view. Figure $5(b)$ is a hidden line display of the multiplicative scaling factors $1 / c(r)$, normalised to unity at and outside the radius of the required field of view, specified as $9.0 \mathrm{~cm}$ in this case.

The approach described here is attractive in that it implies only a simple scaling of the reconstructed image on a point by point basis. However, the correct application of this method depends upon the proper normalisation of the reconstructed image. A background subtraction must be made before modifying the image in order to avoid incorrect scaling of the zero level.

\section{Experimental tests}

The two approaches outlined in the previous section have been implemented for a rotating positron camera (Jeavons et al 1983), and tested by imaging a variety of different phantoms. Positron annihilation events are stored off-line in list mode to allow reprocessing of the data according to the reconstruction method chosen. Three different methods were examined:

(1) The data, with $\phi$ constrained to the range $\pm 4.5^{\circ}$ for each position of the detectors, were backprojected with unit weights. The resulting image was filtered in frequency space with a filter calculated according to the technique described in Schorr et al (1983).

(2) The data were backprojected, without a constraint on $\phi$, using the weights for an $18 \mathrm{~cm}$ field of view (figure $4 \mathrm{c}$ ). The backprojection was then filtered with the same filter as for method (1).

(3) The data were backprojected, again without a constraint on $\phi$, but with unit weights as for method ( 1 ). After filtering, the resulting image was then multiplied, plane by plane, with the scale factors shown graphically in figure $5(b)$.

All images were reconstructed with the same tomographic filter (Schorr et al 1983) and Hanning window function to allow comparisons to be made. In each case, a $16 \mathrm{~mm}$ thick, central transverse plane through the phantom is displayed as a $128 \times 128$ matrix with a pixel size of $2 \mathrm{~mm} \times 2 \mathrm{~mm}$, and all images are normalised to their maximum value.

The phantoms, of which two examples will be presented here, contained $10-20 \mathrm{MBq}$ of ${ }^{68} \mathrm{Ga}$, eluted from an in-house ${ }^{68} \mathrm{Ge}$ generator. Continuous rotation was approximated in practice by collecting data at each of 20 angles with a step of $9^{\circ}$ between detector positions. At every angle, all detected events were stored until a specified number of counts (typically 10000 ) satisfying both $\theta$ and $\phi$ constraints were acquired. This condition is equivalent to an equal data collection time (corrected for isotope decay) at each angle, as required by the three reconstruction methods. The number of collected events varied from angle to angle, and the total number was different for each phantom. The resulting count densities were typically 80000 counts $/ \mathrm{ml}$.

\subsection{Ring phantom}

A plastic tube with a circular cross-section of diameter $1 \mathrm{~cm}$ was filled with ${ }^{68} \mathrm{Ga}$ and fixed in the form of a ring of external diameter $15 \mathrm{~cm}$. Three small cylindrical sources, each of diameter $1.5 \mathrm{~cm}$ and height $5 \mathrm{~cm}$, were positioned inside the ring, as shown in figure $6(a)$. The sources contain the same specific activity $\left(1.5 \mathrm{MBq} \mathrm{ml}^{-1}\right)$ as the ring. The phantom was placed vertically in the camera, such that the plane containing the 


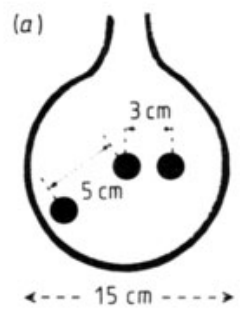

(b)

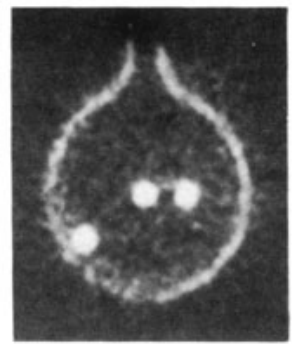

(d)

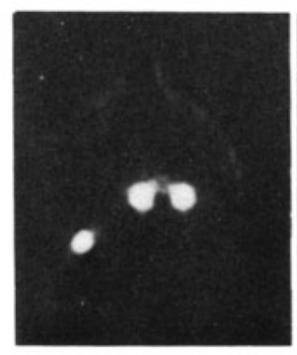

(c)
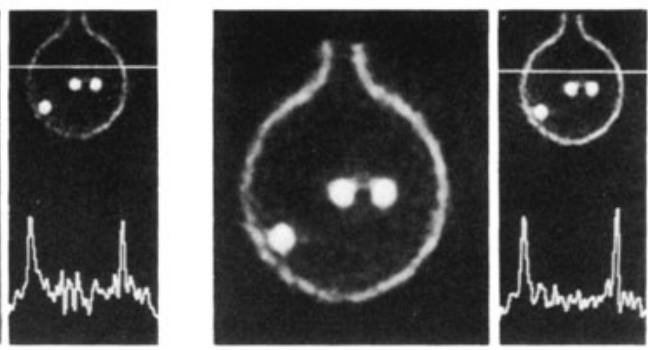

(e)

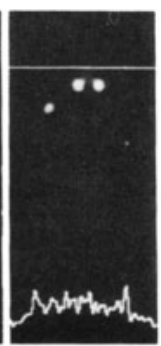

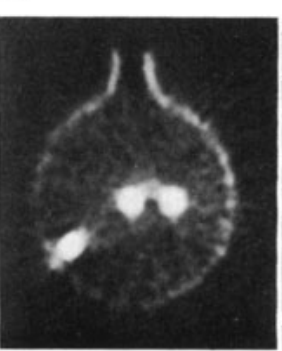

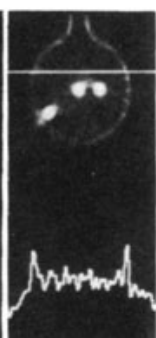

Figure 6. The reconstructed image of a phantom comprising a ring of activity and three small sources each $1.5 \mathrm{~cm}$ in diameter. The images were obtained with the positron camera (Jeavons el al 1983) and a 20-angle rotation. The angular step was $9^{\circ}$. A profile at the level indicated is shown to the right of each image. (a) Sketch of the phantom showing relevant dimensions. (b) Reconstruction with a $\phi$ constraint of $9^{\circ}$ corresponding to a $15 \mathrm{~cm}$ allowable field of view. The image contains 200000 counts. (c) Result of reconstructing without a $\phi$ constraint, but using the weighted backprojection approach described in $\$ 2.1$. (d) Reconstruction without a $\phi$ constraint for the same data as in (b). The image contains 483000 counts. (e) As for $(d)$, except that the reconstruction has been scaled with the weights shown graphically in figure $5(b)$, according to the method described in $\$ 2.2$.

ring was perpendicular to the axis of rotation. A total of 820000 events were collected at 20 angles, with an average random coincidence rate of $33 \%$. The data were reconstructed according to the three methods described above, with an angular constraint on $\theta$ of $\pm 9.3^{\circ}$, thus defining the height of the field of view as $10 \mathrm{~cm}$.

The results are presented in figure 6 . The reconstruction using procedure (1) is shown in figure $6(b)$; the image contains 200000 counts, the remaining 620000 having been eliminated by the angular constraints. Relaxing the constraint on $\phi$, backprojecting with weights, and deconvolving, results in the image shown in figure $6(c)$, which contains 483000 counts; 337000 counts are eliminated by the remaining constraint on $\theta$. The image obtained by relaxing the constraint on $\phi$, backprojecting with unit weights and deconvolving is shown in figure $6(d)$. The result of scaling figure $6(d)$, with the 
factors shown graphically in figure $5(b)$, appears in figure $6(e)$, corresponding to reconstruction procedure (3); this image also contains 483000 counts.

\subsection{Phantom of five sources}

A phantom was constructed consisting of five small sources, each $1.5 \mathrm{~cm}$ in diameter and containing different activities of ${ }^{68} \mathrm{Ga}$. The sources were distributed throughout a $20 \mathrm{~cm}$ diameter field of view, as shown in figure $7(a)$. The phantom was placed in the camera with the plane containing the sources orthogonal to the axis of rotation. A total of 920000 counts was collected at 20 angles with an average random coincidence rate of $30 \%$. The data were reconstructed according to method (2), weighted backprojection, with a $\pm 9.3^{\circ}$ constraint on $\theta$. The result, shown in figure $7(b)$, contains 520000 counts; the circle represents a $20 \mathrm{~cm}$ diameter field of view centred at the axis of rotation.
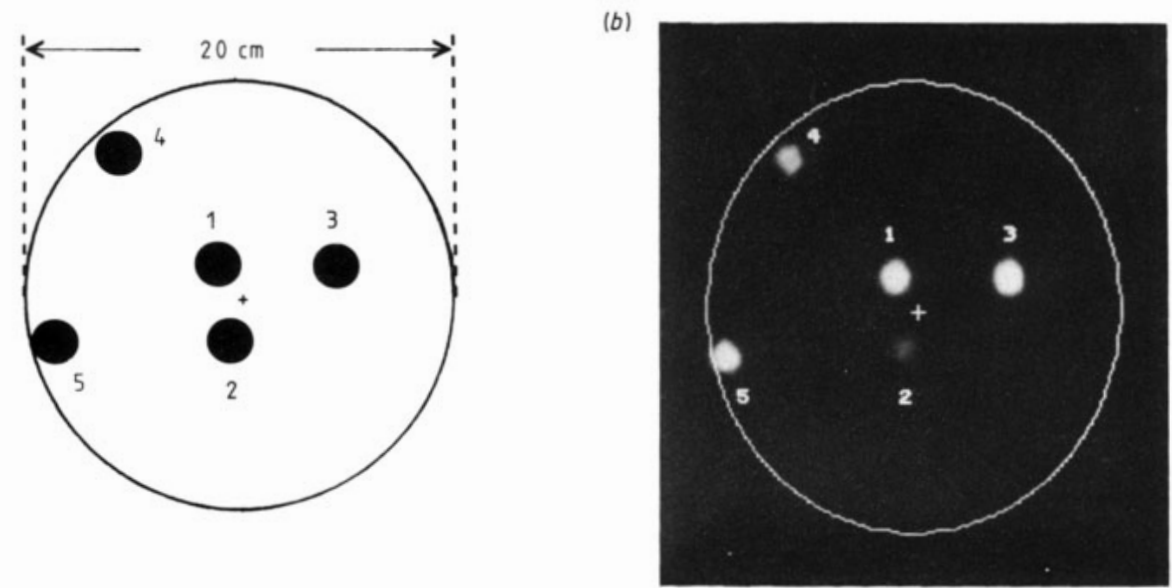

Figure 7. A central plane through a phantom consisting of five small sources each $1.5 \mathrm{~cm}$ diameter, containing differing activities of ${ }^{6 x} \mathrm{Ga}$. (a) Sketch of the phantom. (b) Image taken with 20 angle rotation and reconstructed by weighted backprojection according to the method described in $\$ 2.1$. The image contains 510000 counts; the boundary indicated by the circle has a diameter of $20 \mathrm{~cm}$. The slight elliptic appearance of the boundary is a distortion due to the display.

Defining five regions of interest, one for each of the sources, the average number of reconstructed events per voxel within each region was determined together with an estimate of the standard deviation on the mean value. Since the specific activity of the sources could be measured independently, the mean count density in each region was

Table 1. Mean count densities (with standard deviations) for the sources shown in figure 7.

\begin{tabular}{lllll}
\hline $\begin{array}{l}\text { Source } \\
\text { no. }\end{array}$ & $\begin{array}{l}\text { Distance } \\
\text { from centre } \\
(\mathrm{mm})\end{array}$ & $\begin{array}{l}\text { Specific } \\
\text { activity } \\
\left(\mathrm{MBq} \mathrm{ml}^{-1}\right)\end{array}$ & $\begin{array}{l}\text { Mean count } \\
\text { density } \\
\text { (counts/voxel) }\end{array}$ & $\begin{array}{l}\text { (Counts/voxel }) \\
\left.\mathrm{MBq} \mathrm{m}^{-1}\right)\end{array}$ \\
\hline 1 & 20 & 3.3 & $185 \pm 3$ & $56 \pm 1$ \\
2 & 18 & 1.5 & $84 \pm 3$ & $56 \pm 2$ \\
3 & 50 & 3.3 & $197 \pm 4$ & $60 \pm 1$ \\
4 & 90 & 2.6 & $143 \pm 2$ & $55 \pm 1$ \\
5 & 97 & 3.2 & $184 \pm 4$ & $58 \pm 1$ \\
\hline
\end{tabular}


normalised, for the purposes of comparison, to a source activity of $1 \mathrm{MBq} \mathrm{ml}^{-1}$; the results are summarised in table 1.

\section{Discussion}

The phantom described in $\$ 3.1$ was imaged in order to compare the three different reconstruction methods. To simplify this comparison, the design was chosen so as to minimise scatter and attenuation, and the imaging was performed at count densities somewhat higher than those usually encountered in clinical imaging situations. Similar considerations also apply to the second phantom, described in $\$ 3.2$ which was imaged in order to investigate the uniformity of response of the weighted backprojection method throughout the field of view.

It is evident from comparison of figures $6(b)$ and $(c)$ that weighted backprojection (figure $6 c$ ) offers an improvement in image quality over the conventional reconstruction (figure $6 b$ ) with a constraint on $\phi$. This improvement is most clearly seen on the profiles where the reduction in the fluctuations from noise is due to the increase in statistics which resulted from removing the $\phi$ constraint. The ring does not occupy the full thickness of the section and thus appears less active than the three sources in all images.

It may also be concluded by comparing figures $6(b),(c)$ and $(e)$ that post-reconstruction scaling is at best no better, and may even be slightly worse, than conventional reconstruction with a $\phi$ constraint. Although the noise fluctuations in the central part of the image are reduced by the increase in statistics, the overall signal to noise ratio is decreased for the scaled image compared with the conventional reconstruction. The reasons for this lie not only in the problem of correct normalisation that arises with the post-reconstruction scaling method, but also in the fact that the scale factors amplify both signal and noise equally. Thus, the radial noise patterns characteristic of these reconstructions become increasingly evident towards the edge of the image, where the benefit of the extra statistics is least. This causes the ring activity to appear rather non-uniform. The approximation in equation (2) leads to geometrical distortions in the sources, particularly towards the edge of the field of view (figure $6 e$ ). With weighted backprojection, the ring activity is more uniform, and the geometrical distortions do not arise.

Examination of figure 7 and table 1 shows that, with weighted backprojection, the uniformity of the image is preserved right out to the edge of the field of view. For 20 step rotation, the valid field of view is only $15 \mathrm{~cm}$ according to the conditions given in $\S 2$. In practice, however, the effects of small gaps in the support of the point response function at positions outside the valid field of view are insignificant when compared with typical statistical fluctuations in the data. Source 5 , which is at $9.7 \mathrm{~cm}$ from the centre of rotation contains, within statistical errors, the same count density as source 1 , which is only $2 \mathrm{~cm}$ from the centre. Figure 7 demonstrates that the camera has a uniform response throughout a $20 \mathrm{~cm}$ diameter, circular field of view in a transverse plane.

The advantage of a reconstruction method that does not require a constraint on $\phi$ is summarised in figure 8 . The sensitivity for a $10 \mathrm{~cm}$ field of view $\left(f_{1}\right)$ with the appropriate $\phi$ constraint has been normalised to unity. Enlarging the field to $15.4 \mathrm{~cm}$ $\left(f_{2}\right)$, the figure shows that the sensitivity reduces to $46.5 \%$ of that of the $10 \mathrm{~cm}$ field. That is, an additional $53.5 \%$ of the data would have to be rejected to maintain shift invariance in each angular position for the larger field. However, without the $\phi$ 


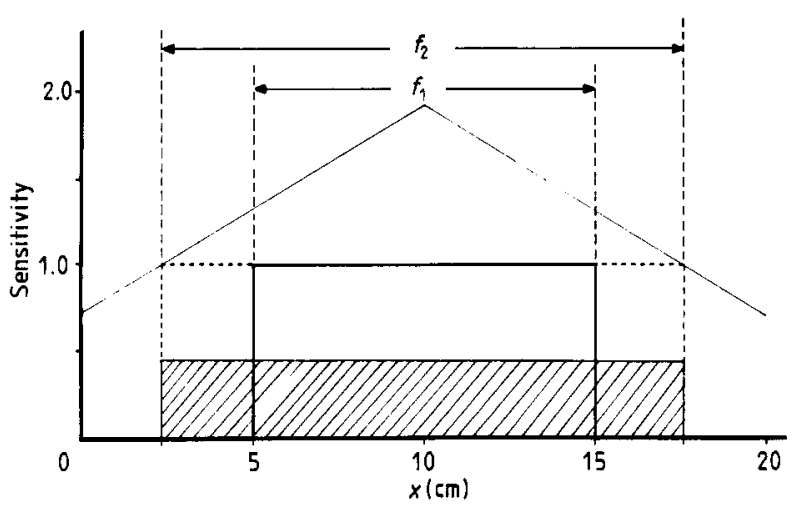

Figure 8. A plot showing the field of view $f_{2}$ that has the same sensitivity without a $\phi$ constraint as the $10 \mathrm{~cm}$ diameter field of view $f_{1}$ with a $\phi$ constraint. The sensitivity for the enlarged field of view with a $\phi$ constraint is also shown (hatched area). Sensitivity is normalised to that of the $10 \mathrm{~cm}$ field of view. $D=20 \mathrm{~cm}$; $Z=61 \mathrm{~cm}$.

constraint, the sensitivity is always that of the upper line, independent of the diameter of the required field of view. Note that the curve peaks at the centre of the field $(x=10)$ where the solid angle is greatest. Comparison of the upper line with the shaded region shows that the increase in sensitivity is considerable for larger fields of view.

\section{Conclusion}

This paper has discussed two approaches towards improving the sensitivity and enlarging the useful field of view for a rotating positron camera by removing the constraint on $\phi$. Weighted backprojection appears superior in that the camera response is more uniform and the images are less noisy than for the post-reconstruction scaling method. Fourier deconvolution reconstruction may be retained since the response function of the overall system is still shift invariant.

In a typical imaging situation, the increase in sensitivity may be as much as a factor of 2.5, which represents a significant improvement when considering the poor statistics of nuclear medicine images. As a consequence of decoupling the sensitivity from the diameter of the field of view, transverse sections through internal organs with dimensions approaching those of the detectors may therefore be accurately imaged with good sensitivity. The weighted backprojection method is now applied in routine clinical imaging (Frey et al 1984).

\section{Acknowledgments}

We wish to thank Dr Benno Schorr, DD Division, CERN, Geneva, for his critical reading of the manuscript. This project is supported by the Fonds National Suisse grant numbers $3.849-0.79,3.986-0.80$ and $3.981-1.80$.

\section{References}

Bateman J E, Connelly J F, Stephenson R and Flesher A C 1980 Nucl. Instrum. Methods Phys. Res. 17783 Chu G and Tam K C 1977 Phys. Med. Biol. 22245

Del Guerra A, Perez-Mendez V, Schwartz G and Nelson W R 1983 IEEE Trans. Nucl. Sci. NS-30 646-51

Frey P, Townsend D, Jeavons A and Donath A 1984 J. Nucl. Med. submitted for publication 
Jeavons A, Hood K, Herlin G, Parkman C, Townsend D, Magnanini R, Frey P and Donath A 1983 IEEE Trans. Nucl. Sci. NS-30 640-5

Jeavons A, Schorr B, Kull K, Townsend D, Frey P and Donath A 1981 Proc. Conf. Medical Radionuclide Imaging (Heidelberg) 1980 (Vienna: IAEA) p49

McKee B T A et al 1979 Proc. 5th Int. Conf. Positron Annihilation (Lake Yamanaka, Japan) 1979 (Japan: Japan Institute of Metals) p413

Ott R J, Bateman J E, Flesher A C, Flower M A, Leach M O, Webb S, Khan O and McCready V R 1983 Br. J. Radiol. 56773

Schorr B and Townsend D 1981 Phys. Med. Biol. 26 305-12

Schorr B, Townsend D and Clack R 1983 Phys. Med. Biol. 28 1009-19

Townsend D, Clack R, Magnanini R, Frey P, Donath A, Schorr B, Jeavons A and Froidevaux A 1983 IEEE Trans. Nucl. Sci. NS-30 p594-600

Townsend D, Frey P, Donath A, Clack R, Schorr B and Jeavons A 1984 Nucl. Inst. Meth. 221105 